\title{
Tuning the performance of aqueous organic redox flow batteries from first principles
}

\author{
Junting Yu, ${ }^{1}$ Tian-Shou Zhao, ${ }^{2}$ and Ding $\operatorname{Pan}^{1,3,4, *}$ \\ ${ }^{1}$ Department of Physics, Hong Kong University \\ of Science and Technology, Hong Kong, China \\ ${ }^{2}$ Department of Mechanical and Aerospace Engineering, \\ Hong Kong University of Science and Technology, \\ Clear Water Bay, Kowloon, Hong Kong, China \\ ${ }^{3}$ Department of Chemistry, Hong Kong University \\ of Science and Technology, Hong Kong, China \\ ${ }^{4}$ HKUST Fok Ying Tung Research Institute, Guangzhou, China
}

(Dated: September 14, 2020)

\begin{abstract}
Aqueous organic redox flow batteries have many appealing properties in the application of largescale energy storage. The large chemical tunability of organic electrolytes shows great potential to improve the performance of flow batteries. Computational studies at the quantum-mechanics level are very useful to guide experiments, but in previous studies explicit water interactions and thermodynamic effects were ignored. Here, we applied the computational electrochemistry method based on ab initio molecular dynamics to calculate redox potentials of quinones and their derivatives. The calculated results are in excellent agreement with experimental data. We mixed side chains to tune their reduction potentials, and found that solvation interactions and entropy effects play a significant role in side-chain engineering. Based on our calculations, we proposed several highperformance negative and positive electrolytes. Our first-principles study paves the way towards the development of large-scale and sustainable electrical energy storage.
\end{abstract}




\section{INTRODUCTION}

The development of renewable energy sources, such as solar and wind, to replace current fossil fuels is a very pressing task to enable sustainable development. A big challenge of integrating solar or wind power into electrical grids is the mismatch between electricity supply and demand, which can be solved by scalable electrical energy storage [1]. To buffer the volatility of solar and wind energy, redox-flow batteries have shown many appealing features including scalability, decoupled energy and power output, high depth of discharging and rapid responsiveness [2]. In addition, if water is used as a solvent to dissolve electrolytes, the safety of flow batteries is greatly enhanced because water is nonflammable and has an unusually large heat capacity.

Since 1970s, the vanadium redox flow battery has been extensively studied and is considered as the closest to wide commercialization [3, 4]. However, the low abundance, high cost, and toxicity of vanadium impede its further application in large-scale energy storage [5, 6]. Recently organic electrolytes have emerged as promising alternatives to vanadium in aqueous flow batteries [7]. Particularly, quinones and their derivatives, such as benzoquinones (BQ), naphthoquinones (NQ), and anthraquinones (AQ) in Fig. 1, show many appealing properties as redox couples [8-12]. They have no rare metal elements, do not need precious metal catalysts, and have great potential for large-scale industrial production. Their large chemical tunability make it possible to optimize their performance. In addition, retired organic flow batteries do not need heavy metal removal, and may be disposed by supercritical water oxidation, a well established green chemistry technology [13].

The performance of organic flow batteries largely depend on three important properties of electrolytes: redox potential, solubility, and chemical stability. Molecular engineering of side chains is an effective method to tailor the three properties of quinones and their derivatives. For example, Huskinson et al. showed that the open circuit voltage increases by $11 \%$ by adding two -OH groups to 9,10-anthraquinone-2,7-disulfonic acid (AQDS) [8]. Most of 
current experimental studies are limited to some halogen, $-\mathrm{OH}$, or $-\mathrm{SO}_{3} \mathrm{X}(\mathrm{X}=\mathrm{H}, \mathrm{Na})$ functionalized quinones, but there are hundreds of thousands of possible quinone/hydroquinone pairs [14]. Computational methods at the quantum mechanics level can be used to evaluate the performance of quinones. Er et al. used a high-throughput computational approach to study the redox potential and solubility of quinones, which are either singly or fully substituted by one functional group [14]. Tabor et al. used a combination of density functional theory (DFT) and semi-empirical calculations to investigate the chemical stability of quinones [15]. In those fast screening methods, water was treated as an implicit medium and thermodynamic effects were ignored. For some functionalized quinone structures, the experimental and predicted redox potentials differ considerably [10].

Here, we chose a variety of functionalized quinone couples with potentially good solubility and chemical stability, and applied ab initio molecular dynamics (AIMD) simulations to calculate their redox potentials. Water molecules were simulated at the atomic level and no empirical parameter was used. The calculated results agree very well with experimental values for existing quinones. We mixed side chains to tune the redox potential, and found that when there are multiple side-chain substitutions, our AIMD results differ from those obtained by the previous model, indicating that explicit water and entropy effects are important in side-chain engineering. We predicted five low-potential negolytes (negative electrolytes) and four high-potential posolytes (positive electrolytes). Our work sheds new light on improving the performance of aqueous organic flow batteries.

\section{RESULTS AND DISCUSSION}

The standard reduction potential of the quinone/hydroquinone $\left(\mathrm{Q} / \mathrm{H}_{2} \mathrm{Q}\right)$ couple versus the standard hydrogen electrode (SHE) is given by $E^{0}=-\frac{\Delta G^{0}}{2 F}$, where $F$ is the Faraday constant and $\Delta G^{0}$ is the Gibbs free energy of the two-electron two-proton reaction:

$$
\mathrm{Q}(\mathrm{aq})+\mathrm{H}_{2}(\mathrm{~g}) \rightarrow \mathrm{H}_{2} \mathrm{Q}(\mathrm{aq})
$$


We applied the computational electrochemistry method based on AIMD and thermodynamic integration [16-18] (see the supporting information for computational details) to calculate $E^{0}$ for quinones with explicit water molecules using the BLYP [19, 20], PBE [21], and HSE06 [22, 23] exchange-correlation functionals. In Fig. 2, we compared our calculated results with experimental data. Both BLYP and PBE functionals underestimate $E^{0}$, and the PBE values are better than BLYP ones. The HSE06 functional gives the best result, whose mean absolute error with respect to experimental data is $0.035 \mathrm{~V}$, similar to the standard deviation $(\sim 0.02 \mathrm{~V})$ from our AIMD simulations. It is known that the generalized gradient approximation (GGA) functionals have a larger charge localization error than the hybrid functionals [24], so HSE06 describes the charge-state change in the redox reaction (1) better than BLYP or PBE. In the following part, we mainly used the HSE06 functional to calculate electronic structure.

Note that the standard potential $E^{0}$ is obtained at $\mathrm{pH}=0$. According to the Nernst equation, the reduction potential in reaction (1) as a function of $\mathrm{pH}$ is [25]:

$$
E=E^{0}-\frac{k_{B} T \ln 10}{e} p H,
$$

where $k_{B}$ is the Blotzmann constant, $\mathrm{T}$ is the temperature, and $e$ is the elementary charge, so the measured potential should decrease linearly with $\mathrm{pH}$. However, the experimental Pourbaix diagram in Fig. 3 seems to contain several line segments. The reason is that how the redox reaction happens depends on pH. Fig. 3 shows the possible reaction steps involving quinones. We need the dissociation constant of the two reactions below to judge the actual redox reaction:

$$
\begin{aligned}
& p K_{a 1}: \mathrm{H}_{2} \mathrm{Q}(\mathrm{aq}) \rightarrow \mathrm{HQ}^{-}(\mathrm{aq})+\mathrm{H}^{+}(\mathrm{aq}) \\
& p K_{a 2}: \mathrm{HQ}^{-}(\mathrm{aq}) \rightarrow \mathrm{Q}^{2-}(\mathrm{aq})+\mathrm{H}^{+}(\mathrm{aq})
\end{aligned}
$$

The acid dissociation constant is given by

$$
p K_{a}=-\log _{10} \frac{\left[X^{-}\right]\left[H^{+}\right]}{[H X]}=p H-\log _{10} \frac{\left[X^{-}\right]}{[H X]},
$$


where $\mathrm{X}^{-}$denotes $\mathrm{HQ}^{-}$or $\mathrm{Q}^{2-}$ and $\mathrm{HX}$ is $\mathrm{H}_{2} \mathrm{Q}$ or $\mathrm{HQ}^{-}$. According to Pauling's rule for oxoacids, successive $p K_{a}$ values usually increase, so $p K_{a 2}$ is larger than $p K_{a 1}$. When the solution is very acidic and $\mathrm{pH}$ is smaller than $p K_{a 1}$, the concentration of $\mathrm{H}_{2} \mathrm{Q}$ is higher than that of $\mathrm{HQ}^{-}$or $\mathrm{Q}^{2-}$, so the main reduction reaction is

$$
\mathrm{Q}(\mathrm{aq})+2 \mathrm{H}^{+}(\mathrm{aq})+2 \mathrm{e}^{-} \rightarrow \mathrm{H}_{2} \mathrm{Q}(\mathrm{aq}) .
$$

When $\mathrm{pH}$ is between $p K_{a 1}$ and $p K_{a 2}$, the concentration of $\mathrm{HQ}^{-}$is higher than that of $\mathrm{H}_{2} \mathrm{Q}$ or $\mathrm{Q}^{2-}$, so the main reduction reaction becomes

$$
\mathrm{Q}(\mathrm{aq})+\mathrm{H}^{+}(\mathrm{aq})+2 \mathrm{e}^{-} \rightarrow \mathrm{HQ}^{-}(\mathrm{aq})
$$

When $\mathrm{pH}$ is larger than $p K_{a 2}, \mathrm{Q}^{2-}$ will be the dominating electrolyte in the solution, so the main reduction reaction is

$$
\mathrm{Q}(\mathrm{aq})+2 \mathrm{e}^{-} \rightarrow \mathrm{Q}^{2-}(\mathrm{aq})
$$

If in a reduction reaction there are $m$ protons removed and $n$ electrons transferred, the pH-dependent reduction potential in Eq. (2) becomes

$$
E=E^{0}-\frac{m k_{B} T \ln 10}{n e} p H
$$

so the slopes in the Pourbaix diagram are $-\frac{k_{B} T \ln 10}{e},-\frac{k_{B} T \ln 10}{2 e}$, and 0 corresponding to reactions (6), (7), and (8), respectively.

We take AQDS as an example and performed first-principles simulations to calculate the Pourbaix diagram as shown in Fig. 3. $E^{0}$ in reactions (6), (7), and (8) was calculated as $0.245,0.010$, and $-0.302 \mathrm{~V}$, respectively. The calculated $p K_{a 1}$ and $p K_{a 2}$ are 8.363 and 10.525, respectively (see the supporting information), so our calculated Pourbaix diagram constains three segments: $\mathrm{pH}<8.363,8.363<\mathrm{pH}<10.525$, and $\mathrm{pH}>10.525$, and the line slopes are - $0.060 \mathrm{~V} / \mathrm{pH},-0.030 \mathrm{mV} / \mathrm{pH}$, and 0 , respectively. Fig. 3 shows that the calculated curve agrees very well with the experimental result, which together with the results in Fig. 2 gives us confidence to study a variety of quinones and derivatives. 
We apply side-chain engineering to tailor the redox potential of quinone derivatives. The reduction potential of parent quinones has the following order: $B Q>N Q>A Q$, so it is often to choose $\mathrm{AQ}$ as the backbone of negolytes and BQ as the backbone of posolytes. We considered 5 common side chains: $-\mathrm{SO}_{3} \mathrm{H}$, $-\mathrm{PO}_{3} \mathrm{H}_{2}$, $-\mathrm{COOH},-\mathrm{OH}$, and $-\mathrm{NH}_{2}$, which were predicted to help to dissolve quinones in water [14]. The first three are electron withdrawing groups (EWGs), and the last two are electron donating groups (EDGs). Generally, we found that EWGs increase $E^{0}$, while EDGs decease $E^{0}$. Besides, the position of EDGs strongly affects $E^{0}$. When EDGs are next to the $\mathrm{C}=\mathrm{O}$ group, $E^{0}$ decreases more. Our findings are consistent with previous studies [14]. There are no experimental data for the $-\mathrm{PO}_{3} \mathrm{H}_{2}$, $-\mathrm{COOH}$, and $-\mathrm{NH}_{2}$ substitutions. Our AIMD simulations show that the $-\mathrm{PO}_{3} \mathrm{H}_{2}$ group increases $E^{0}$ slightly more than the $-\mathrm{SO}_{3} \mathrm{H}$ and $-\mathrm{COOH}$ groups, and the $-\mathrm{NH}_{2}$ group decreases $E^{0}$ more than $-\mathrm{OH}$, which were not reported in previous theoretical studies.

In previous calculations [14], $E^{0}$ was calculated by a linear function

$$
E^{0}=-\frac{\Delta H_{f}}{n F}+b
$$

where $\Delta H_{f}$ is the DFT total energy change of reaction (1) in the gas phase, and $b$ is a constant fitted from the experimental $E^{0}$ data of parent quinones. In Fig. 4, we compared the gas-phase model results with our AIMD ones; the mean absolute error is $0.097 \mathrm{~V}$, nearly three times as large as that between the experimental and HSE06 results as shown in Fig. 2. Particularly when there are multiple side chain substitutions, the difference becomes larger (see also Tables SI and SII in the supporting information). Note that only parent quinones without side chains were used to fit Eq. (10) [8, 14], so side chain effects may not be well captured.

In the gas-phase model calculation, we first relax the molecular structure and then calculate the DFT energy. Side chains have freedom to rotate and vibrate, but the structural relaxation often can only find a local minimum, so depending on the initial configuration, $E^{0}$ obtained by Eq. 10 may be very different for quinone isomers. For example, by varying the 
orientation of the $\mathrm{O}-\mathrm{H}$ bonds in 1,8-dihydroxyanthraquinone ( $\mathrm{DH}(1,8) \mathrm{AQ}$, dantron), $E^{0}$ can change by as large as $0.560 \mathrm{~V}$. We may try to find the global minimum structure in the gas phase by starting from different initial configurations, but because of the presence of water, the obtained structure may not be the stable one in the solution. At finite temperature, the rotation and vibration of side chains in water contribute to the entropy part of the free energy of quinones, which, as well as solvation interactions, is included in AIMD simulations, but not in the gas-phase model. Our results suggest that they are important to determining $E^{0}$. A great advantage of quinones is that we can engineer side chains to optimize their performance. In the case of multiple side-chain substitutions, a general computational method which can properly take into account the solvation interactions and entropy contributions of side chains is essential to making accurate predictions.

We first mixed the EDGs and EWGs to functionalize AQ to work as negolytes. To increase the open-circuit voltage, we look for quinones whose reduction potentials are close to $0 \mathrm{~V}$, but $E^{0}$ can not be negative; otherwise the $\mathrm{H}_{2}$ gas will be produced in the charging process. We put EDGs away from the $\mathrm{C}=\mathrm{O}$ groups in $\mathrm{AQ}$, because previous experimental studies suggest that if EDGs are too close, the solubility is poor [10]. Fig. 5 shows 5 negolyte candidates with potentially high performance, whose $E^{0}$ is between 0 and $0.1 \mathrm{~V}$.

It is more challenging to find suitable organic posolytes, because molecules with high reduction potential are usually not chemically stable in aqueous solutions; for example, as electron deficient molecules, they may react with water via nucleophilic addition [10, 15, 26]. Here, to enhance chemical stability, we replace all the $\mathrm{C}-\mathrm{H}$ groups adjacent to the $\mathrm{C}=\mathrm{O}$ groups that are susceptible to oxidation with $\mathrm{C}-\mathrm{PO}_{3} \mathrm{H}_{2}$ or $\mathrm{C}-\mathrm{COOH}$ groups, as shown in Fig. 6. The reduction potentials of fully substituted BQ are slightly lower than $1 \mathrm{~V}$, and similar to that of 1,2-benzoquinone-3,5-disulfonic acid (1,2-BQDS(3,5)) reported in the previous experimental study [9] and subject to the Michael reaction [26]. Interestingly, if there are two $-\mathrm{PO}_{3} \mathrm{H}_{2}$ group substitutions in 1,2-benzoquinone-3,5-diphosphonic acid(1,2$\operatorname{BQDP}(3,5))$, the reduction potential can be as high as $1.051 \mathrm{~V}$, which means that $E^{0}$ does 
not increase monotonically with the number of EWGs. Through the Michael reaction, the C-H groups on the ring of $1,2-\mathrm{BQDP}(3,5)$ or $1,2-\mathrm{BQDS}(3,5)$ may be substituted by the C$\mathrm{OH}$ groups, which will lower their reduction potentials. Thus, there is a trade off between reduction potential and chemical stability in designing organic posolytes using quinones.

\section{CONCLUSION}

We applied the computational electrochemistry method based on ab initio molecular dynamics and thermodynamical integration to calculate the redox potential of quinones and their derivatives. The calculated standard reduction potentials and the theoretical Pourbaix diagram are in excellent agreement with experimental results. We compared our method with the gas-phase model used in previous studies, and found that when there are multiple side-chain substitutions, our AIMD results may differ considerably from those obtained by the previous model, indicating that solvation interactions and entropy effects are very important in side-group engineering of quinones. Based on our calculations, we proposed five low- $E^{0}$ negolytes and four high- $E^{0}$ posolytes.

The large chemical tunability of quinones show great potential to improve the performance of aqueous organic redox flow batteries. The computational method showed here may be used to select high-performance electrolyte candidates and speed up the trial-and-error process in experiments. Our first-principles study paves the way towards the development of large-scale and sustainable electrical energy storage.

\section{METHODS}

\section{Ab initio molecular dynamics}

We performed ab intio molecular dynamics (AIMD) simulations using the CP2K Quickstep package [27, 28]. We used Goedecker-Teter-Hutter (GTH) norm-conserving pseudopo- 
tentials to describe core electrons $[29,30]$ and a triple- $\zeta$ doubly polarized (TZV2P) basis set to expand the Kohn-Sham orbitals of valence electrons. The electron density cutoff is 340 Ry. We used deuterium instead of hydrogen to make use of a larger time step, which is 0.5 fs. There are one quinone molecule and $49 \sim 58$ water molecules in a cubic simulation box with periodic boundary conditions (see Table SI and SII in the supporting information). In canonical ensemble (i.e., NVT) simulations, the temperature is $330 \mathrm{~K}$, controlled by the Bussi-Donadio-Parrinello thermostat $(\tau=100 \mathrm{fs})[31]$. We applied the BLYP exchange-correlation functional and the D3 van der Waals correction [32] to generate AIMD trajectories [33], which were used in the following PBE+D3 and HSE06+D3 calculations for the total energies of reactants and products in thermodynamic integration (TI). Each trajectory is at least $5 \mathrm{ps}$, and in the TI calculations, vertical energy gaps were calculated every 100 AIMD steps. The convergence tests are shown in the supporting information. In HSE06 hybrid functional calculations, we used the auxiliary density matrix method (ADMM) [34] to reduce computational costs and the auxiliary basis set is FIT3.

\section{Classical molecular dynamics}

We performed classical molecular dynamics simulations using the GROMACS package [35] to prepare initial configurations for AIMD. We used the Gromos 54A7 force field [36] and the SPC/E water model [37]. The force field parameters for quinone molecules were generated by the Automated force field Topology Builder (ATB) [38]. We built the simulation box by doubling the box lengths along the $\mathrm{x}, \mathrm{y}, \mathrm{z}$ directions in AIMD simulations. We

carried out $1 \mathrm{~ns}$ NPT simulations at $\mathrm{P}=1$ bar to get the size of simulation boxes, and then 500 ps NVT simulations before AIMD simulations. 


\section{DATA AVAILABILITY}

The data that support this study are in the supporting information and available upon request from the authors.

\section{ACKNOWLEDGEMENTS}

The authors thank Qing Chen and Rongbiao Tong for their helpful discussions. D.P. acknowledge support from the Croucher Foundation through the Croucher Innovation Award, and the Energy Institute in Hong Kong University of Science and Technology. T.S. Zhao acknowledges support from the Research Grants Council of the Hong Kong Special Administrative Region, China (Project No. T23-601/17-R). This work was carried out using computational resources from the National Supercomputer Center in Guangzhou, China.

* dingpan@ust.hk

[1] Gür, T. M. Review of electrical energy storage technologies, materials and systems: challenges and prospects for large-scale grid storage. Energy Environ. Sci. 11, 2696-2767 (2018).

[2] Alotto, P., Guarnieri, M. \& Moro, F. Redox flow batteries for the storage of renewable energy: A review. Renew. Sustain. Energy Rev. 29, 325-335 (2014).

[3] Leung, P. et al. Progress in redox flow batteries, remaining challenges and their applications in energy storage. RSC Adv. 2, 10125-10156 (2012).

[4] Kear, G., Shah, A. A. \& Walsh, F. C. Development of the all-vanadium redox flow battery for energy storage: a review of technological, financial and policy aspects. Int. J. Energy Res. 36, 1105-1120 (2012).

[5] Barnhart, C. J. \& Benson, S. M. On the importance of reducing the energetic and material demands of electrical energy storage. Energy Environ. Sci. 6, 1083-1092 (2013). 
[6] Ghosh, S. K., Saha, R. \& Saha, B. Toxicity of inorganic vanadium compounds. Res. Chem. Intermed. 41, 4873-4897 (2015).

[7] Chen, H., Cong, G. \& Lu, Y.-C. Recent progress in organic redox flow batteries: Active materials, electrolytes and membranes. J. Energy Chem. 27, 1304-1325 (2018).

[8] Huskinson, B. et al. A metal-free organic-inorganic aqueous flow battery. Nature 505, 195 (2014).

[9] Yang, B., Hoober-Burkhardt, L., Wang, F., Surya Prakash, G. K. \& Narayanan, S. R. An inexpensive aqueous flow battery for large-scale electrical energy storage based on watersoluble organic redox couples. J. Electrochem. Soc. 161, A1371-A1380 (2014).

[10] Wedege, K., Drazevic, E., Konya, D. \& Bentien, A. Organic redox species in aqueous flow batteries: Redox potentials, chemical stability and solubility. Sci. Rep. 6, 39101 (2016).

[11] Gerhardt, M. R. et al. Anthraquinone derivatives in aqueous flow batteries. Adv. Energy Mater. 7, 1601488 (2017).

[12] Yang, Z. et al. Alkaline benzoquinone aqueous flow battery for large-scale storage of electrical energy. Adv. Energy Mater. 8, 1702056 (2018).

[13] Li, Y. \& Wang, S. Supercritical water oxidation for environmentally friendly treatment of organic wastes. In Advanced Supercritical Fluids Technologies (IntechOpen, 2019).

[14] Er, S., Suh, C., Marshak, M. P. \& Aspuru-Guzik, A. Computational design of molecules for an all-quinone redox flow battery. Chem. Sci. 6, 885-893 (2015).

[15] Tabor, D. P. et al. Mapping the frontiers of quinone stability in aqueous media: implications for organic aqueous redox flow batteries. J. Mater. Chem. A 7, 12833-12841 (2019).

[16] Cheng, J., Sulpizi, M. \& Sprik, M. Redox potentials and pka for benzoquinone from density functional theory based molecular dynamics. J. Chem. Phys. 131, 154504 (2009).

[17] Cheng, J., Liu, X., VandeVondele, J., Sulpizi, M. \& Sprik, M. Redox potentials and acidity constants from density functional theory based molecular dynamics. Acc. Chem. Res. 47, 3522-3529 (2014). 
[18] Cheng, J. \& VandeVondele, J. Calculation of electrochemical energy levels in water using the random phase approximation and a double hybrid functional. Phys. Rev. Lett. 116, 086402 (2016).

[19] Becke, A. D. Density-functional exchange-energy approximation with correct asymptotic behavior. Phys. Rev. A 38, 3098-3100 (1988).

[20] Lee, C., Yang, W. \& Parr, R. G. Development of the colle-salvetti correlation-energy formula into a functional of the electron density. Phys. Rev. B 37, 785-789 (1988).

[21] Perdew, J. P., Burke, K. \& Ernzerhof, M. Generalized gradient approximation made simple. Phys. Rev. Lett. 77, 3865-3868 (1996).

[22] Heyd, J., Scuseria, G. E. \& Ernzerhof, M. Hybrid functionals based on a screened coulomb potential. J. Chem. Phys. 118, 8207-8215 (2003).

[23] Heyd, J., Scuseria, G. E. \& Ernzerhof, M. Erratum: "hybrid functionals based on a screened coulomb potential" [j. chem. phys. 118, 8207 (2003)]. J. Chem. Phys. 124, 219906 (2006).

[24] Mori-Sánchez, P., Cohen, A. J. \& Yang, W. Localization and delocalization errors in density functional theory and implications for band-gap prediction. Phys. Rev. Lett. 100, 146401 (2008).

[25] Lower, S. Chem1 virtual textbook: Electrochemistry (2020). https://chem.libretexts.org/Bookshelves/General_Chemistry/.

[26] Yang, B. et al. High-performance aqueous organic flow battery with quinone-based redox couples at both electrodes. J. Electrochem. Soc. 163, A1442-A1449 (2016).

[27] Hutter, J., Iannuzzi, M., Schiffmann, F. \& VandeVondele, J. cp2k: atomistic simulations of condensed matter systems. Wiley Interdiscip. Rev. Comput. Mol. Sci. 4, 15-25 (2014).

[28] VandeVondele, J. et al. Quickstep: Fast and accurate density functional calculations using a mixed gaussian and plane waves approach. Comput. Phys. Commun. 167, $103-128$ (2005).

[29] Goedecker, S., Teter, M. \& Hutter, J. Separable dual-space gaussian pseudopotentials. Phys. Rev. B 54, 1703-1710 (1996). 
[30] Hartwigsen, C., Goedecker, S. \& Hutter, J. Relativistic separable dual-space gaussian pseudopotentials from H to Rn. Phys. Rev. B 58, 3641-3662 (1998).

[31] Bussi, G., Donadio, D. \& Parrinello, M. Canonical sampling through velocity rescaling. J. Chem. Phys. 126, 014101 (2007).

[32] Grimme, S., Antony, J., Ehrlich, S. \& Krieg, H. A consistent and accurate ab initio parametrization of density functional dispersion correction (DFT-D) for the 94 elements h-pu. J. Chem. Phys. 132, 154104 (2010).

[33] Seitsonen, A. P. \& Bryk, T. Melting temperature of water: DFT-based molecular dynamics simulations with D3 dispersion correction. Phys. Rev. B 94, 184111 (2016).

[34] Guidon, M., Hutter, J. \& VandeVondele, J. Auxiliary density matrix methods for hartree-fock exchange calculations. J. Chem. Theory Comput. 6, 2348-2364 (2010).

[35] Berendsen, H., van der Spoel, D. \& van Drunen, R. Gromacs: A message-passing parallel molecular dynamics implementation. Comput. Phys. Commun. 91, 43 - 56 (1995).

[36] Schmid, N. et al. Definition and testing of the gromos force-field versions 54a7 and 54b7. Eur. Biophys. J. 40, 843 (2011).

[37] Berendsen, H. J. C., Grigera, J. R. \& Straatsma, T. P. The missing term in effective pair potentials. J. Phys. Chem. 91, 6269-6271 (1987).

[38] Malde, A. K. et al. An automated force field topology builder (ATB) and repository: Version 1.0. J. Chem. Theory Comput. 7, 4026-4037 (2011).

[39] Johnsson Wass, J. R. T., Ahlberg, E., Panas, I. \& Schiffrin, D. J. Quantum chemical modeling of the reduction of quinones. J. Phys. Chem. A 110, 2005-2020 (2006). 


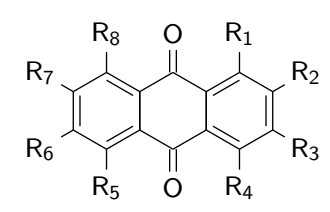

$9,10-\mathrm{AQ}$
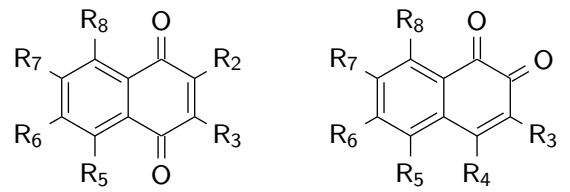

$1,4-N Q$

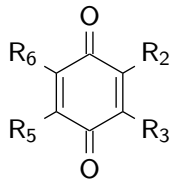

$1,4-B Q$

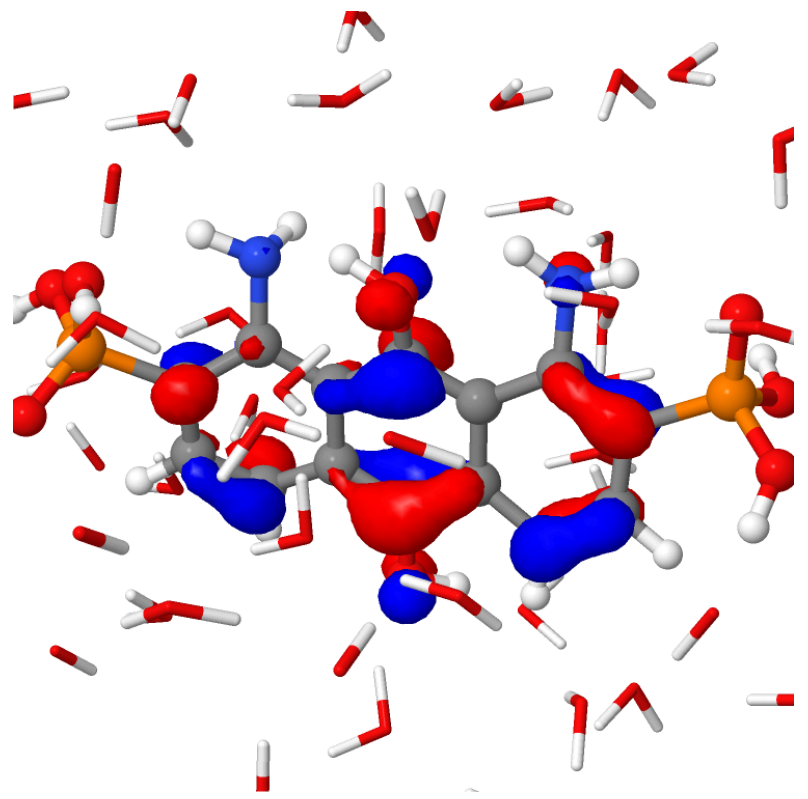

(b)

FIG. 1. Molecular structures of quinones. (a) Chemical structures of 9,10-anthraquinone $(9,10-$ AQ), 1,4-naphthoquinone (1,4-NQ), 1,2-naphthoquinone (1,2-NQ), 1,4-benzoquinone (1,4-BQ), and 1,2-benzoquinone (1,2-BQ). (b) the highest occupied molecular orbital of 1,8-diamino-9,10anthrahydroquinone-2,7-diphosphoric acid (DN(1,8)AHQDP $(2,7))$ dissolved in water. 


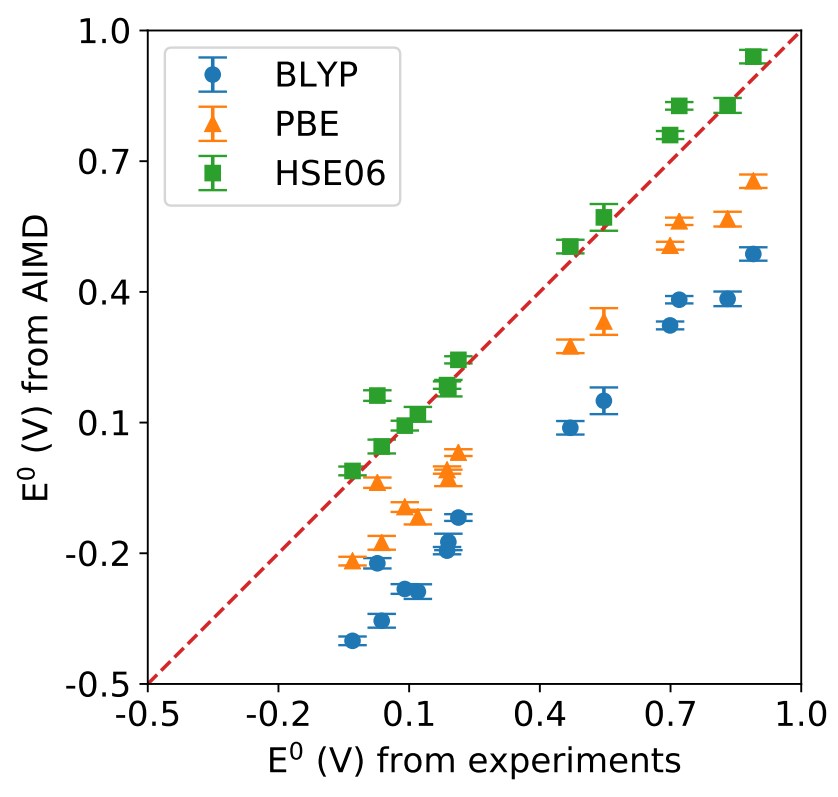

FIG. 2. Comparison of the standard reduction potentials $\left(E^{0}\right)$ calculated by ab initio molecular dynamics (AIMD) simulations versus experimental values . The AIMD results were obtained using three exchange-correlation functionals: BLYP, PBE, and HSE06. The experimental data are from Refs. $[8,10,11,26,39]$. The mean absolute error between the experimental and HSE06 results is $0.035 \mathrm{~V}$. 


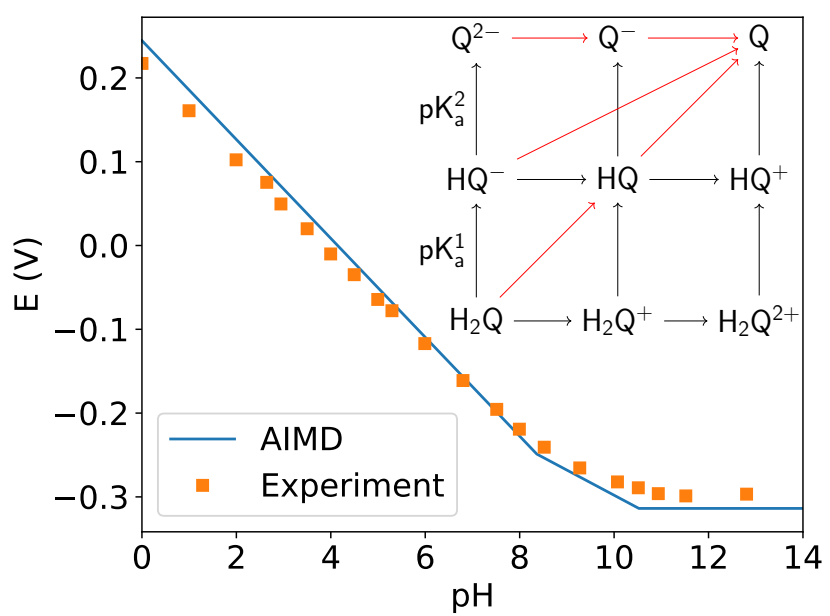

FIG. 3. Pourbaix diagram of 9,10-anthraquinone-2,7-disulfonic acid (AQDS). The AIMD result is compared with the experimental data from Ref. [8]. The embedded figure shows the scheme of squares for quinone reactions. The horizontal arrows indicate oxidation reactions and the vertical arrows are for deprotonation reactions. The red arrows correspond to three line segments in the $E$ vs. $p H$ plot.

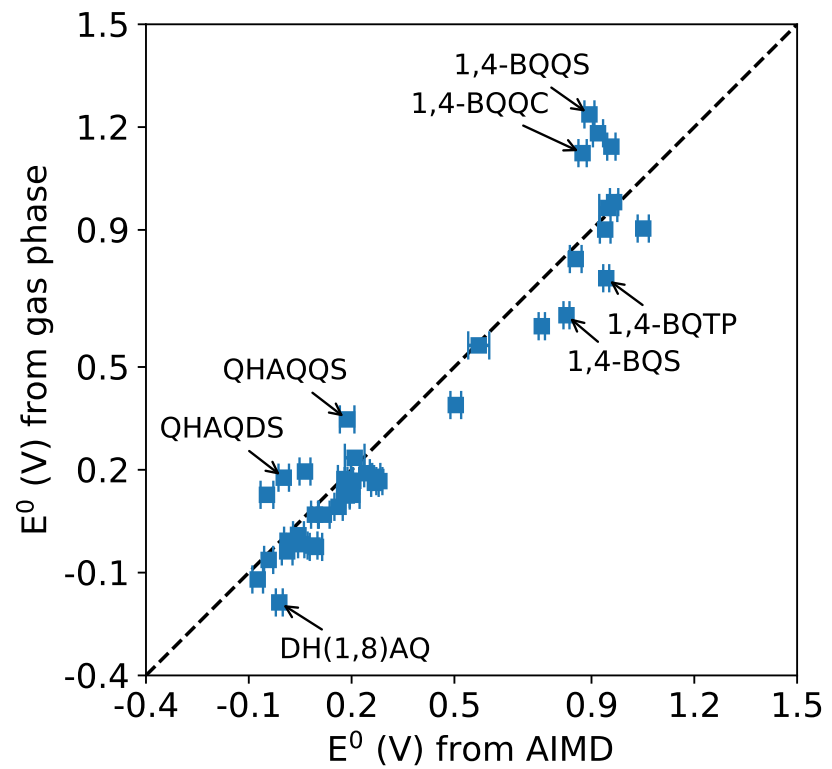

FIG. 4. Comparison of the standard reduction potentials $\left(E^{0}\right)$ calculated by AIMD simulations and the gas-phase model method $[8,14]$. The mean absolute error is $0.097 \mathrm{~V}$. 

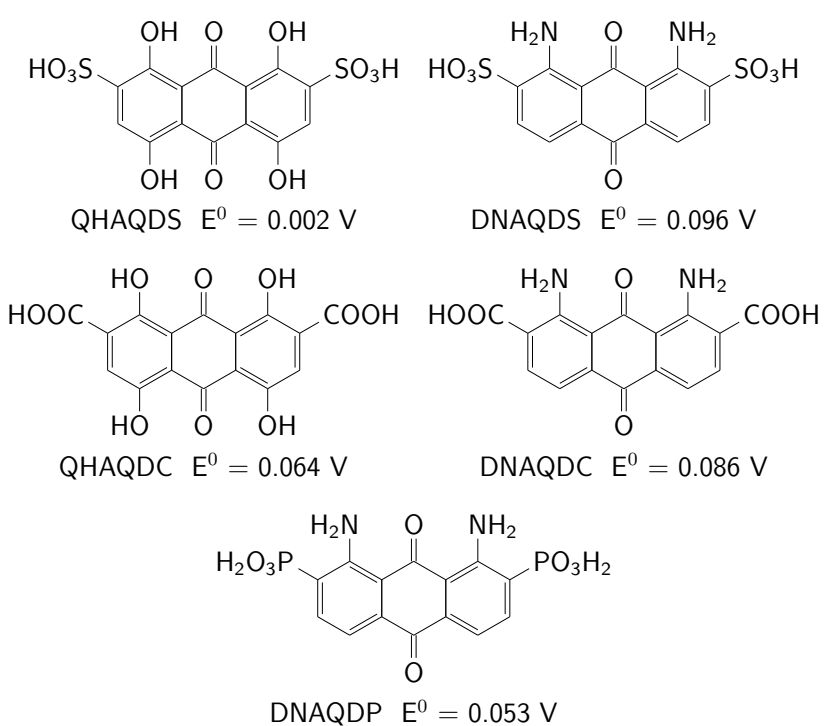

FIG. 5. Proposed molecules as negative electrolytes. According to AIMD simulations, their standard reduction potentials are close to $0 \mathrm{~V}$.
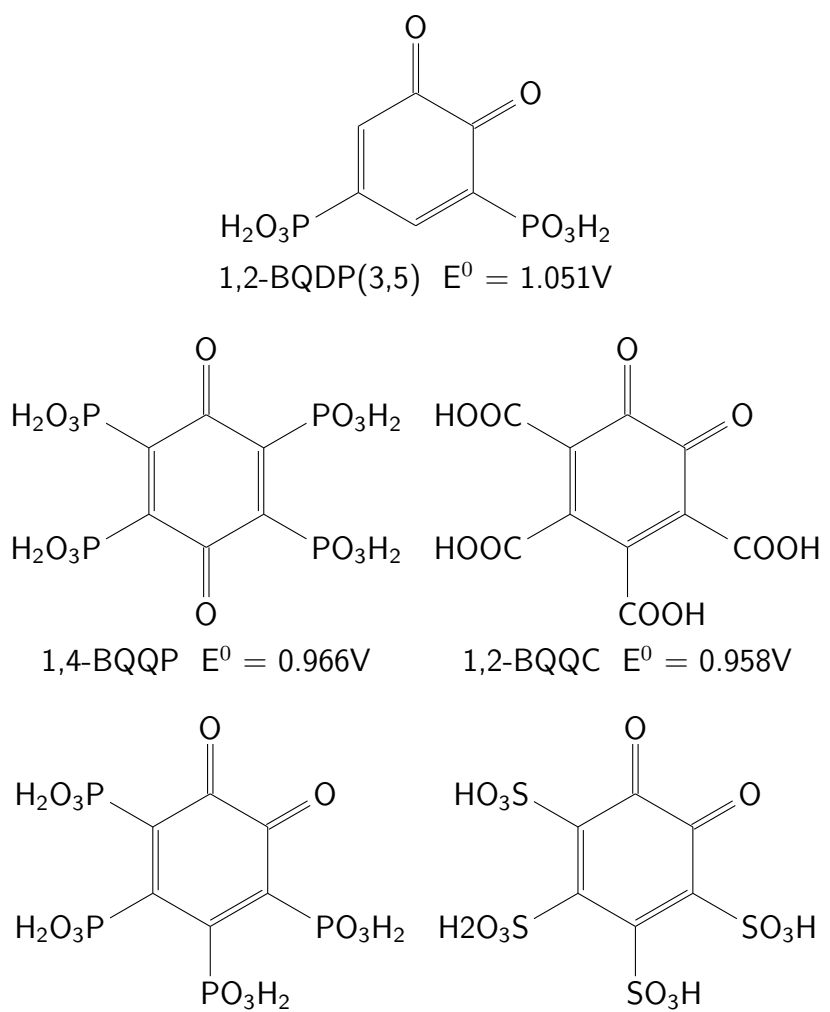

$1,2-B Q Q P \quad E^{0}=0.949 \mathrm{~V}$

$1,2-\mathrm{BQQS} \quad \mathrm{E}^{0}=0.919 \mathrm{~V}$

FIG. 6. Proposed molecules as positive electrolytes. According to AIMD calculations, they have high standard reduction potentials. 